\title{
First results from ideal 2-D MHD reconstruction: magnetopause reconnection event seen by Cluster
}

\author{
W.-L. Teh and B. U. Ö. Sonnerup \\ Dartmouth College, Hanover, New Hampshire 03755, USA \\ Received: 15 April 2008 - Revised: 3 July 2008 - Accepted: 31 July 2008 - Published: 12 September 2008
}

\begin{abstract}
We have applied a new reconstruction method (Sonnerup and Teh, 2008), based on the ideal single-fluid MHD equations in a steady-state, two-dimensional geometry, to a reconnection event observed by the Cluster-3 (C3) spacecraft on 5 July 2001, 06:23 UT, at the dawn-side NorthernHemisphere magnetopause. The event has been previously studied by use of Grad-Shafranov (GS) reconstruction, performed in the deHoffmann-Teller frame, and using the assumption that the flow effects were either negligible or the flow was aligned with the magnetic field. Our new method allows the reconstruction to be performed in the frame of reference moving with the reconnection site (the X-line). In the event studied, this motion is tailward/equatorward at $140 \mathrm{~km} / \mathrm{s}$. The principal result of the study is that the new method functions well, generating a magnetic field map that is qualitatively similar to those obtained in the earlier GSbased reconstructions but now includes the reconnection site itself. In comparison with the earlier map by Hasegawa et al. (2004), our new map has a slightly improved ability ( $\mathrm{cc}=0.979$ versus $\mathrm{cc}=0.975$ ) to predict the fields measured by the other three Cluster spacecraft, at distances from $\mathrm{C} 3$ ranging from $2132 \mathrm{~km}(\mathrm{C} 1)$ to $2646 \mathrm{~km}$ (C4). The new field map indicates the presence of a magnetic X-point, located some $5300 \mathrm{~km}$ tailward/equatorward of $\mathrm{C} 3$ at the time of its traversal of the magnetopause. In the immediate vicinity of the $\mathrm{X}$-point, the ideal-MHD assumption breaks down, i.e. resistive and/or other effects should be included. We have circumvented this problem by an ad-hoc procedure in which we allow the axial part of convection electric field to be nonconstant near the reconnection site. The new reconstruction method also provides a map of the velocity field, in which the inflow into the wedge of reconnected field lines and the plasma jet within it can be seen, and maps of the electric potential and of the electric current distribution. Even though
\end{abstract}

Correspondence to: W.-L. Teh

(wai-leong_teh@Dartmouth.edu) the velocity map is expected to be inaccurate near the $\mathrm{X}$ point, it provides high-quality predictions $(\mathrm{cc}=0.969)$ of the velocity components at points along the path of $\mathrm{C} 1$, some of which are close to the X-point; the predictions of density and pressure are less good. Except near the reconnection site, the new reconstruction provides a complete characterization, in unprecedented detail, of the entire dynamic plasma and field equilibrium, reconstructed from the $\mathrm{C} 3$ data. It represents our best prediction to date of what the actual configuration was like. But, since substantial time variations were present in the event, the recovered structure by necessity includes considerable time aliasing. The invariant direction used in the reconstruction, is found to agree, within $6^{\circ}$, with a recent theoretical prediction of the $\mathrm{X}$-line orientation by Swisdak and Drake (2007).

Keywords. Magnetospheric physics (Magnetopause, cusp, and boundary layers) - Space plasma physics (Kinetic and MHD theory; Magnetic reconnection)

\section{Introduction}

The term Grad-Shafranov (GS) reconstruction refers to a method of producing maps of two-dimensional, timeindependent field and plasma structures, governed by a GSlike equation, from data taken by one or more spacecraft traversing the structures. It was originally developed (Sonnerup and Guo, 1996; Hau and Sonnerup, 1999) for application to ideal magneto-hydrostatic structures, which are governed by the classical GS equation, but has recently been generalized to include the effects of magnetic-field aligned plasma flow, and to recover streamlines in flow transverse to a unidirectional field (Sonnerup et al., 2006a). Both of these cases are governed by GS-like equations. Applications of magneto-hydrostatic reconstruction have allowed the recovery of a variety of magnetopause structures (Hau and

Published by Copernicus Publications on behalf of the European Geosciences Union. 


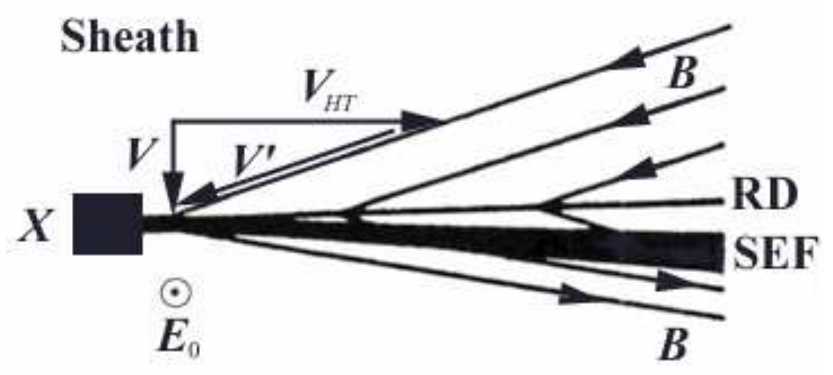

Sphere

Fig. 1. Schematic drawing illustrating the difference between the deHoffmann-Teller (HT) frame and the proper frame (the frame of the drawing) for an asymmetric two-dimensional reconnection configuration at the magnetopause. This configuration (only half of which is shown) contains an upstream rotational discontinuity (RD) followed by a slow-mode expansion fan (SEF) (Levy et al., 1964). In the HT frame, which moves to the right with speed $V_{H T}$ relative to the proper frame, the plasma flow, $V^{\prime}$, crossing the RD is along the magnetic field so that the tangential electric field in the $\mathrm{RD}$, including the component $E_{z}$, vanishes at locations well away from the SEF and the reconnection site (the X-point). In the HT frame, the SEF structure appears to be moving downward, away from the RD, while the reconnection site is moving to the left: the global configuration is time dependent. In the proper frame, the entire reconnection configuration is time stationary and anchored to the $\mathrm{X}$. The inflow velocity is $V$ and the electric field component $E_{z}$ is nonzero and constant, $E_{z}=E_{0}$, within the entire configuration; $E_{0}$ is the reconnection electric field (from Sonnerup and Teh, 2008).

Sonnerup, 1999; Hu and Sonnerup, 2003a; Sonnerup et al., 2004; Hasegawa et al., 2006; Teh and Hau, 2004, 2007a; Lui et al., 2008), including those in the 5 July 2001 event, where active reconnection occurred (Hasegawa et al., 2004, 2005), as well as flux ropes in the solar wind (e.g. Hu et al. 2001, 2003b) and in the earth's geomagnetic tail (Hasegawa et al., 2007a; Zhang et al., 2007). Recently, an application that includes the effects of field-aligned flow in the 5 July reconnection event has been reported (Teh et al., 2007b) and the streamlines in a Kelvin-Helmholtz (KH) generated vortex train in Earth's low-latitude boundary layer have been reconstructed (Hasegawa et al., 2007b). In both of these applications, the base assumptions of the GS-like reconstruction are being somewhat stretched: For the reconnection event, the reconstruction was performed in the deHoffmann-Teller (HT) frame of reference, in which the flow is as field aligned as the data permit. But, as illustrated in Fig. 1, the reconnection configuration is time dependent, when seen in this frame: The reconnection site (the X-line) is moving rapidly to the left, making it difficult or impossible to recover the plasma/field behavior in its vicinity or to pinpoint its location. Another consequence of the motion is that the various substructures of the reconnection wedge are receding from each other, i.e. the separation between them is increasing with time. But, provided the reconnection rate is small and the observations are made well away from the reconnection site, the resulting time dependence is probably not important for the reconstruction of a substructure such as the rotational discontinuity (RD). In the case of GS-based streamline reconstruction, a difficulty is that, in practice, there are usually remnant magnetic field components in the reconstruction plane. These components may have significant dynamic effects on the flow and may themselves develop observable structures as a result of the flow.

The problems mentioned above can be removed, or at least alleviated, by performing reconstruction that is based directly on the ideal, time-independent, 2-D magneto-hydrodynamic (MHD) equations, rather than being restricted to situations where the equations can be cast in the form of a GS-like system. We have shown that such reconstruction is indeed possible: The basic mathematical theory for it has been developed, along with a numerical code that has been validated by application to an exact solution of the MHD equations (Sonnerup and Teh, 2008). The purpose of the present paper is to present the first results of this general MHD reconstruction, as applied to the magnetopause reconnection event seen by the Cluster spacecraft around 06:23 UT on 5 July 2001. Ideal MHD does not describe conditions in the immediate vicinity of the reconnection site but our application will show that its approximate location can be established and that a detailed overall picture of the reconnection geometry can be obtained. An application of MHD reconstruction to KH activity, observed by one of the THEMIS spacecraft in the low-latitude boundary layer, has also been made and will be reported separately (Eriksson et al., 2008 ${ }^{1}$ ).

The paper is organized as follows. In Sect. 2, the mathematical basis for MHD-based reconstruction is briefly summarized. In Sect. 3, the method is applied to the 5 July 2001, event. As mentioned already, this event has been studied previously, both by use of magneto-hydrostatic GS reconstruction (Hasegawa et al., 2004, 2005) and by use of GS-like reconstruction that incorporates the effects of field-aligned flow (Teh et al., 2007b). The choice of this event allows inter-comparison of results from the new method and those from the earlier ones, which will provide further important validation of the field maps from the previous reconstructions and will also highlight differences in the results caused by the underlying differences in physical assumptions. Section 4 contains further discussion of the results and of the prospects for accurately reconstructing reconnection event in the immediate vicinity of the reconnection site itself, where the assumptions of ideal MHD break down.

\footnotetext{
${ }^{1}$ Eriksson, S., Hasegawa, H., Teh, W.-L., McFadden, J. P., Glassmeier, K.-H., Roux, A., Angelopoulos, V., Sonnerup, B. U. Ö., Cully, C. M., and Ergun, R. E.: Magnetic island formation between large-scale flow vortices at an undulating postnoon magnetopause for northward IMF, J. Geophys. Res., submitted, 2008.
} 


\section{Theory}

A detailed description of how one arrives at the formulas that form the basis of MHD reconstruction has been given by Sonnerup and Teh (2008). For convenience we provide a brief summary of the method in this section. It is based on ideal, single-fluid MHD with the assumption of a steady state and two dimensions, the invariant axis being the z-axis of the coordinate system in which the reconstruction is performed. The xy-plane is called the reconstruction plane. It is in this plane that maps can be generated of magnetic field $\boldsymbol{B}_{\perp}=\left[B_{x}(x, y), B_{y}(x, y)\right]$, flow field $\boldsymbol{v}_{\perp}=\left[v_{x}(x, y), v_{y}(x, y)\right]$, electric current density $j_{\perp}=\left[j_{x}(x, y), j_{y}(x, y)\right]$, electric field $\boldsymbol{E}_{\perp}=\left[E_{x}(x, y), E_{y}(x, y)\right]$, and other quantities such as plasma pressure $p(x, y)$ and density $\rho(x, y)$, as well as axial field $B_{z}(x, y)$, flow $v_{z}(x, y)$, and current density $j_{z}(x, y)$. In a steady state, Faraday's law requires the total axial electric field to be a constant, $E_{z}=E_{0}$, independent of $x$ and $y$. In reconstructions of ongoing reconnection, $E_{0}$ is the reconnection electric field. In regions where ideal, single-fluid MHD and therefore the frozen field condition applies, the total electric field may be replaced by the convection field $\boldsymbol{E}_{c}=-\boldsymbol{v} \times \boldsymbol{B}$. The $\mathrm{x}$-axis of the reconstruction coordinate system is along the spacecraft path through the structure to be recovered; the \pm y-axis is the direction of integration away from the spacecraft path. It is assumed that, in its motion along the $\mathrm{x}$-axis, the spacecraft has measured all quantities required in the reconstruction, namely $[\boldsymbol{B}(x, 0), \boldsymbol{v}(x, 0), p(x, 0), \rho(x, 0)]$. The measured time series of these quantities are converted to spatial variation along the x-axis, by use of the velocity $\boldsymbol{V}_{0}$ of the structure past the observing spacecraft. The determination of this velocity and of the invariant direction, which is perpendicular to it, is discussed at the end of the section.

The magnetic field and flow field in the reconstruction plane are expressed in terms of a vector potential $A(x, y)$ and a compressible stream function $\psi(x, y)$, respectively, so that $\boldsymbol{B}_{\perp}=\nabla A \times \hat{z}$ and $\rho \boldsymbol{v}_{\perp}=\nabla \psi \times \hat{z}$. Field lines projected onto the reconstruction plane are then curves defined by $A(x, y)=$ const. and streamlines are defined by $\psi(x, y)=$ const. The initial values, $A(x, 0)$ and $\psi(x, 0)$, are obtained by integration of the measured $y$-components of field and flow:

$A(x, 0)=\int(\partial A / \partial x) d x=-\int B_{y}(x, 0) d x$

and

$\psi(x, 0)=\int(\partial \psi / \partial x) d x=-\int \rho(x, 0) v_{y}(x, 0) d x$

In the integration of the MHD equations, the vector potential and stream function are advanced by use of the Taylor expansions

$A(x, y \pm \Delta y)=A(x, y) \pm \Delta y \partial A / \partial y+(1 / 2)(\Delta y)^{2} \partial^{2} A / \partial y^{2}(3)$ $\psi(x, y \pm \Delta y)=\psi(x, y) \pm \Delta y \partial \psi / \partial y+(1 / 2)(\Delta y)^{2} \partial^{2} \psi / \partial y^{2}(4)$

The quantities $\left[\rho, p, v_{z}, B_{z}\right]$ are advanced only to lowest order, e.g., $\rho(x, y \pm \Delta y)=\rho(x, y) \pm \Delta y \partial \rho / \partial y$. The unknown derivatives needed to integrate the MHD system can be arranged into the row vector

$$
\begin{aligned}
\boldsymbol{X}= & {\left[(\partial \rho / \partial y),(\partial p / \partial y),\left(\partial v_{z} / \partial y\right),\left(\partial B_{z} / \partial y\right),\right.} \\
& \left.\left(\partial^{2} \psi / \partial y^{2}\right),\left(\partial^{2} A / \partial y^{2}\right)\right]
\end{aligned}
$$

This vector is governed by the matrix equation

$\mathbf{M}_{0} \boldsymbol{X}^{T}=\boldsymbol{Y}_{0}^{T}$

where the matrix $\mathbf{M}_{0}$ is given by

$$
\mathbf{M}_{0}=\left(\begin{array}{cccccc}
-v_{x} v_{y} & 0 & 0 & 0 & v_{y}-B_{y} / \mu_{0} \\
-v_{y}^{2} & 1 & 0 & B_{z} / \mu_{0} & 0 & B_{x} / \mu_{0} \\
0 & 0 & \rho v_{y} & -B_{y} / \mu_{0} & 0 & 0 \\
v_{y} B_{z} / \rho & 0 & B_{y} & -v_{y} & 0 & 0 \\
\left(v_{y} B_{x}-v_{x} B_{y}\right) & 0 & 0 & 0 & B_{y} & -\rho v_{y} \\
-c_{p} / \rho & c_{v} / p & 0 & 0 & 0 & 0
\end{array}\right) \text { (7) }
$$

and the column vector $\boldsymbol{Y}_{0}^{T}$ by

$$
\boldsymbol{Y}_{0}^{T}=\left(\begin{array}{c}
-\partial\left[p+\left(B_{y}^{2}+B_{z}^{2}\right) / 2 \mu_{0}\right] / \partial x-\rho \partial\left(v_{x}^{2} / 2\right) / \partial x \\
-\rho v_{x} \partial v_{y} / \partial x+v_{y} \partial \rho v_{x} / \partial x+\left(B_{x} / \mu_{0}\right) \partial B_{y} / \partial x \\
-\rho v_{x} \partial v_{z} / \partial x+\left(B_{x} / \mu_{0}\right) \partial B_{z} / \partial x \\
-B_{x} \partial v_{z} / \partial x+v_{x} \partial B_{z} / \partial x-\left(v_{x} B_{z} / \rho\right) \partial \rho / \partial x \\
\rho v_{x} \partial B_{x} / \partial x-B_{x} \partial \rho v_{x} / \partial x \\
\rho v_{x} d S / d \psi
\end{array}\right)
$$

The matrix $\mathbf{M}_{0}$ and the vector $\boldsymbol{Y}_{0}^{T}$ contain only quantities and derivatives that are known at each step, i.e. at each y-value, of the integration. The last equation in the set (6) comes from the conservation of entropy $S(\psi)$ along the streamlines. This entropy function, which is assumed to remain valid throughout the integration domain, can be evaluated at points along the spacecraft path (the $\mathrm{x}$-axis), after the stream function $\psi(x, 0)$ has been obtained from Eq. (2). As the integration proceeds, the matrix $\mathbf{M}_{0}$ must be inverted at each grid point. The integration code contains low-pass filtering after each step $\Delta y$ in order to help suppress the spurious and rapidly growing parts of the solution that unavoidably arise in this type of initial-value problem. Even so, the integration domain remains limited to a rectangle with an aspect ratio of typically $0.1-0.2$, with the long sides parallel to the $\mathrm{x}$-axis.

One of the major problems in doing reconstruction of the type discussed here is to find the optimal invariant direction, $\hat{z}$, and the optimal velocity of motion, $\boldsymbol{V}_{0}$, of the coordinate system in which the reconstruction should be performed. In ideal circumstances, both of these vectors can be derived from the condition that, in the proper moving frame, and along the right invariant direction $\hat{z}$, the total electric field $E_{z}$ should be strictly constant. A least-squares procedure for finding the frame velocity and the axis from this requirement, using single-spacecraft data, has been developed and tested by Sonnerup and Hasegawa (2005). Experience has indicated that in some events it gives good results 


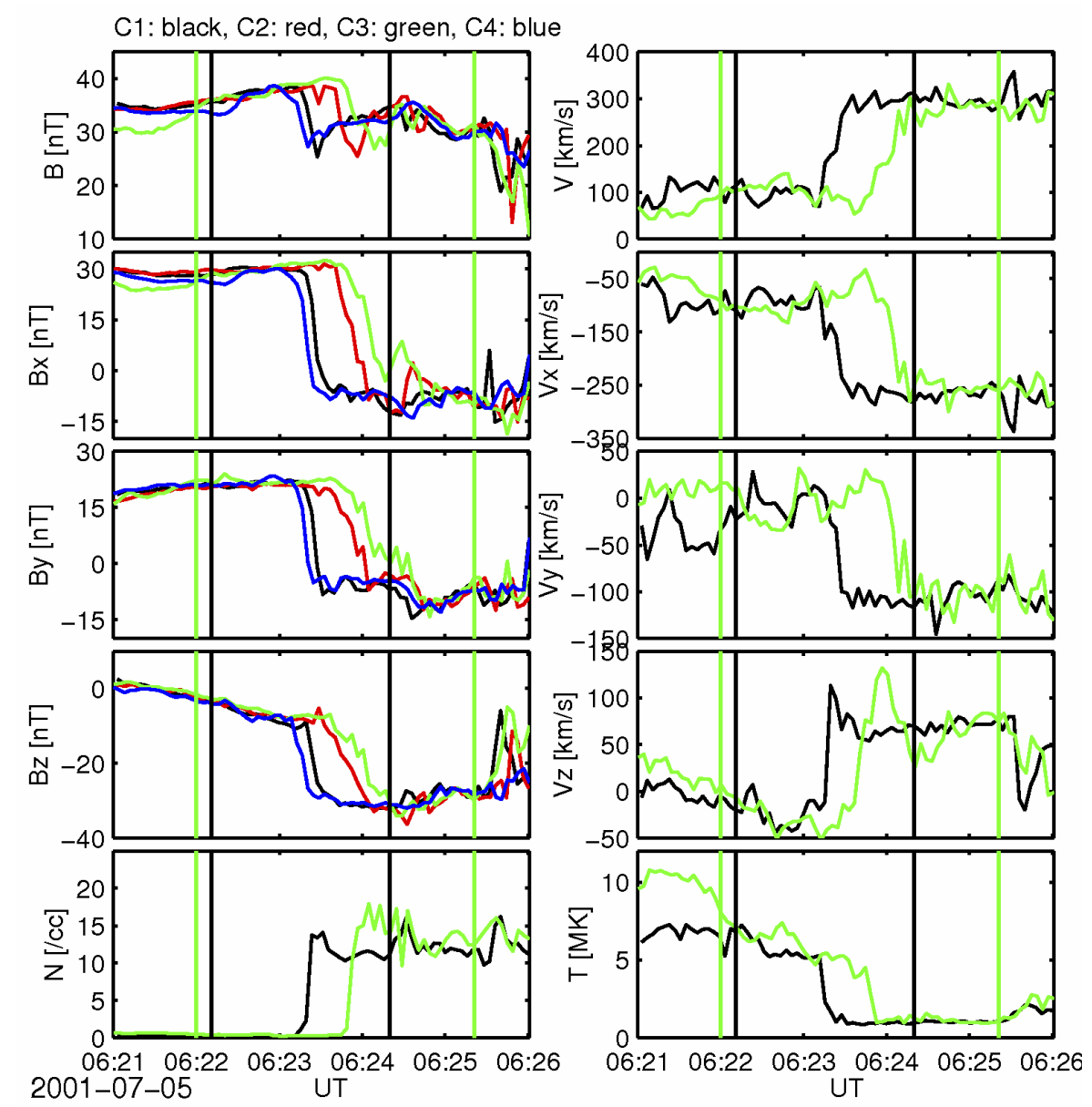

Fig. 2. Time series of Cluster measurements around, and during, a magnetopause crossing on 5 July 2001. The color code is: black=C1; red $=\mathrm{C} 2$; green $=\mathrm{C} 3$; blue $=\mathrm{C} 4$. The left (right) panels show magnetic field (ion velocity) and ion density (temperature), with vector components along the GSE coordinate axes. The plasma data for $\mathrm{C} 1$ and $\mathrm{C} 3$ are from the CIS/HIA instrument. The interval between black (green) vertical lines is used in the reconstruction based on $\mathrm{C} 1$ (C3) data.

but in many others it does not. Other methods for singlespacecraft data may be based on searching for an axis and motion that render the entropy function $S(\psi)$ optimally well organized and consistent for streamlines that are encountered more than once by the spacecraft, i.e., streamlines that intersect the $\mathrm{x}$-axis more than once, during the data interval used in the reconstruction. If four-spacecraft data are available and the spacecraft separation is small compared to the structure being studied, then the tensor representing $\nabla \boldsymbol{B}$ can in principle be used to find the invariant direction (e.g. Shi et al., 2005); for larger separations, multiple reconstructions can be performed, and a trial-and-error procedure used, to optimize the correlation between map prediction and actual measurements, as described by Hasegawa et al. (2004, 2005). Also, Zhou et al. (2006) have developed a multiple triangulation analysis to determine the orientation of a magnetic flux rope, using multi-spacecraft data.

\section{Cluster event on 5 July 2001, at 06:23 UT}

This magnetopause encounter occurred on the dawn-side and in the Northern Hemisphere at $[X, Y, Z]_{\mathrm{GSE}}=[-6.78,-14.97,6.24] R_{E}$. Time series of the relevant measurements are shown in Fig. 2, in which the time intervals used in the reconstruction are indicated by vertical lines, black for $\mathrm{C} 1$ and green for $\mathrm{C} 3$. The transition of the spacecraft is from the magnetosphere to the magnetosheath. The event has been extensively analyzed, both in terms of single and multi-spacecraft determinations of the magnetopause normal vector and motion (Haaland et al., 2004; Sonnerup et al., 2006b, 2007), and in terms of magneto-hydrostatic GS reconstructions performed in the HT frame (Hasegawa et al., 2004, 2005) and a GS-like reconstruction, also in the HT frame, that includes the effects of field-aligned flow (Teh et al., 2007b). The invariant direction (the z-axis) in these reconstructions was determined by optimization of the correlation coefficient between 


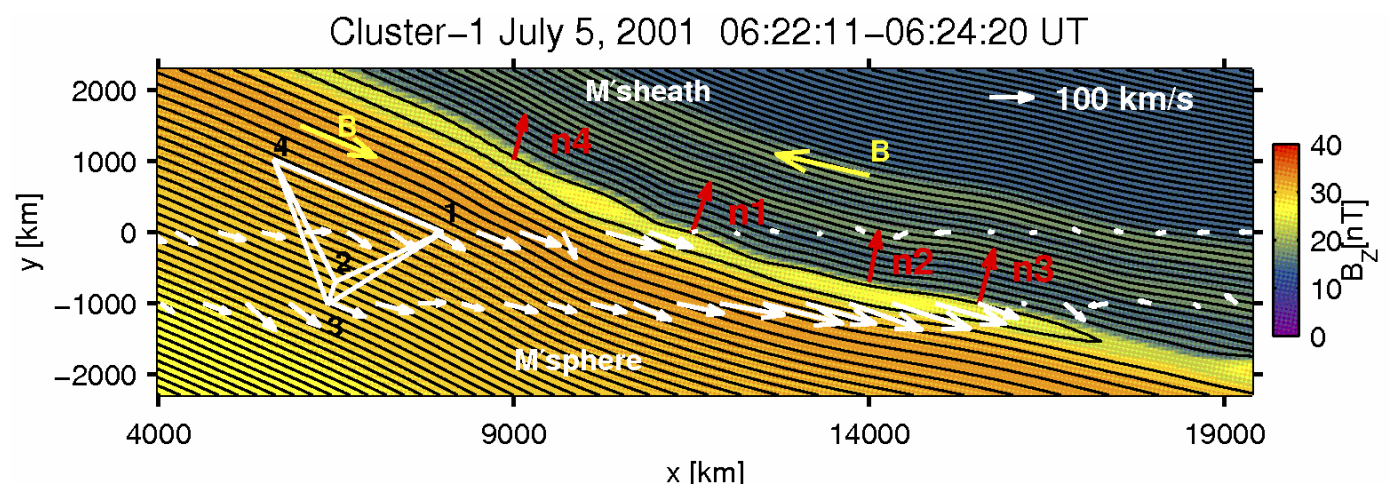

Fig. 3. Portion of magnetic field map from magneto-hydrostatic Grad-Shafranov reconstruction, based on $\mathrm{C} 1$ data taken during the magnetopause crossing on 5 July 2001. The white vectors are plasma velocities in the HT frame, projected onto the reconstruction plane. The red arrows are the normal vectors from MVAB0. The white constellation denotes the projected location of the four Cluster spacecraft on the map. Magnetosphere (magnetosheath) is in lower left (upper right) of the map; sunward/northward is to the right. For a more detailed description, see Hasegawa et al. (2004).

measured and reconstructed field components, as described by Hasegawa et al. (2004).

The most important finding from the earlier reconstructions is that the magnetopause changed its structure in a rather dramatic way in the $30 \mathrm{~s}$ time interval between the crossing by Cluster-1 (C1) and that by Cluster-3 (C3): Regardless of reconstruction method, the $\mathrm{C} 1$ results indicate the crossing of a thin magnetopause layer, containing at most some narrow magnetic islands (see Fig. 3), and predicting that the other three spacecraft should also see such a thin magnetopause layer. However, the C3-based reconstruction showed instead a much thicker layer and the unmistakable signatures of reconnection activity. A wedge-like region containing field lines that appear to cross the magnetopause and provide magnetic connection between its two sides was now present. The flux content in the wedge allowed a lower limit of $0.47 \mathrm{mV} / \mathrm{m}$ to be placed on the average reconnection electric field (Teh et al., 2007b). These authors also found the plasma flow, in the HT frame they employed, to be supersonic in the wedge. On the whole, this event appears to be an example of reconnection onset, in the time interval between the $\mathrm{C} 1$ and the $\mathrm{C} 3$ crossings. In the $30 \mathrm{~s}$ time interval, the Walén slope, evaluated during the magnetopause traversal, increased from 0.57 in the $\mathrm{C} 1$ crossing to 1.03 in the $\mathrm{C} 3$ crossing (Hasegawa et al., 2004). The reconnection site was located somewhat tailward/equatorward of the spacecraft so that the reconnection jet traversed by $\mathrm{C} 3$ was in a direction opposing the main magnetosheath flow. As a consequence, no jet-associated velocity maximum was seen in the spacecraft frame.

The MHD reconstruction in the present study is concerned with the magnetopause crossing by $\mathrm{C} 3$. It uses the same invariant axis $\hat{z}$ as in the papers by Hasegawa et al. (2004) and Teh et al. (2007b) and, for the sole purpose of determining the magnetopause velocity, the same HT-frame velocity,
$\boldsymbol{V}_{H T}$. The GSE location of the crossing and the orientation of the invariant axis relative to the fields on the two sides of the magnetopause are shown in Fig. 4, along with the direction of motion of the X-line. The earlier work used a magnetopause normal vector $\hat{\boldsymbol{n}}$, calculated from constrained magnetic minimum-variance analysis (MVAB0: $\langle\boldsymbol{B}\rangle \cdot \hat{\boldsymbol{n}}=0$ ). Our $\hat{\boldsymbol{n}}$ vector was obtained by instead using the constraint $\hat{\boldsymbol{n}} \cdot \hat{z}=0$; it forms an angle of $4.4^{\circ}$ with the previously used vector. The result of this rotation is that our $\hat{\boldsymbol{n}}$ has a negative average normal field component, $\langle\boldsymbol{B}\rangle \cdot \hat{\boldsymbol{n}}=-2.3 \mathrm{nT}$, and a negative normal flow component, $\left(\langle\boldsymbol{v}\rangle-\boldsymbol{V}_{H T}\right) \cdot \hat{\boldsymbol{n}}=-13.8 \mathrm{~km} / \mathrm{s}$, during the magnetopause traversal (06:23:53-06:24:37 UT). Also, the magnetopause velocity, $\hat{\boldsymbol{n}} \cdot \boldsymbol{V}_{H T}$, decreases from $-56.2 \mathrm{~km} / \mathrm{s}$ (Hasegawa et al., 2004) to $-37.1 \mathrm{~km} / \mathrm{s}$. An important difference is that, in our reconstruction, the frame velocity relative to the spacecraft is no longer $\boldsymbol{V}_{H T}$ but is instead a velocity $\boldsymbol{V}_{0}$, composed of a component $\boldsymbol{V}_{0}^{\prime}$ (see Fig. 4) at right angles to both the invariant axis and the normal vector, plus the vector $\hat{\boldsymbol{n}}\left(\boldsymbol{V}_{H T} \cdot \hat{\boldsymbol{n}}\right)$, the latter describing the magnetopause motion along the normal direction. The method by Sonnerup and Hasegawa (2005) to find the axis and its motion failed to give believable values for this event. For this reason, the axis orientation was taken from the work by Hasegawa et al. (2004), and the magnitude of $\boldsymbol{V}_{0}^{\prime}\left(\left|\boldsymbol{V}_{0}^{\prime}\right|=135 \mathrm{~km} / \mathrm{s}\right)$ was determined by requiring the average axial convection electric field $E_{0}=-\langle\boldsymbol{v} \times \boldsymbol{B}\rangle \cdot \hat{z}$ in the moving frame to be $0.47 \mathrm{mV} / \mathrm{m}$, which is the lower limit calculated by Teh et al. (2007b) from their reconstruction. As illustrated in the left panel of Fig. 5, the standard deviation of the individual convective $E_{z}$ values from this average is $\pm 0.28 \mathrm{mV} / \mathrm{m}$; the average itself is uncertain by $0.08 \mathrm{mV} / \mathrm{m}$. The fluctuations indicate the presence of substantial temporal variations that cannot be captured in the reconstruction. These fluctuations in the initial convection $E_{z}$ data (at $y=0$ ) are removed prior to the start of the integration. This is 


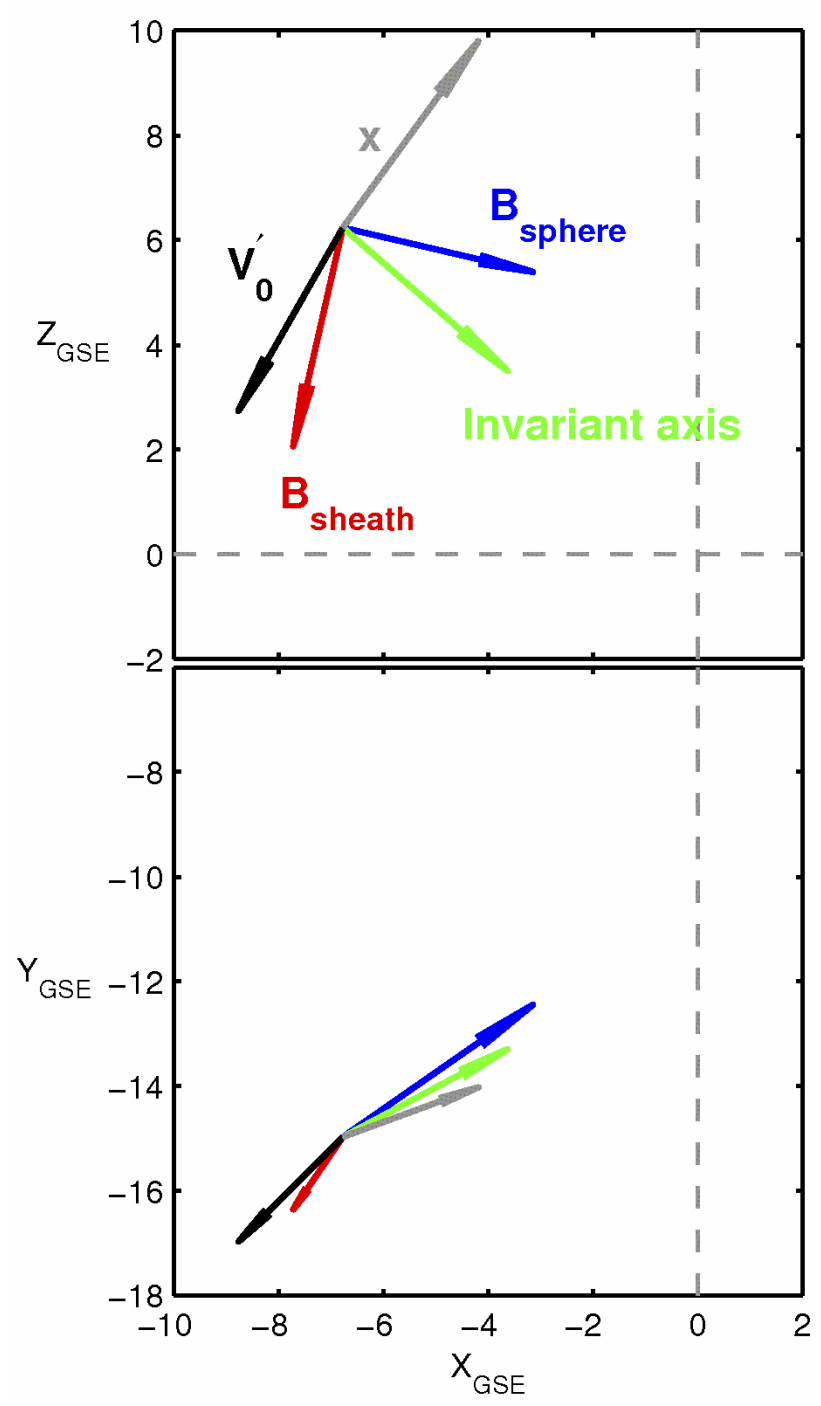

Fig. 4. Schematic of magnetic fields and invariant axis. The figure shows a projection onto the GSE xz-plane (upper panel) and xy-plane (lower panel) of the magnetospheric and magnetosheath fields and the invariant axis (the $\mathrm{X}$-line and its motion along the magnetopause with velocity $\left.\boldsymbol{V}_{0}^{\prime}=[-59.8,-59.9,-105.2] \mathrm{km} / \mathrm{s}\right)$ at the observation site (the point of origin of the vectors) on the dawnside northern magnetopause. Also shown is the $\mathrm{x}$-axis used in the reconstruction.

achieved by making small adjustments in the component of $\boldsymbol{v}_{\perp}(x, 0)$ perpendicular to $\boldsymbol{B}_{\perp}$. In each subsequent step of the integration, the same type of nudging is used to preserve strict constancy of the convective $E_{z}$ throughout the integration domain, except near the reconnection site. The procedure used there will be discussed presently.

The entropy function $S(\psi)$, obtained in our new reconstruction frame for C3, is shown in the right panel of Fig. 5, in which the data points are the values of $S$, calculated directly from the measured density and temperature. The high entropy on the left in the figure represents magnetospheric conditions; the lower level on the right corresponds to magnetosheath conditions. The wedge of reconnected field lines occupies the central portion of the plot, where the entropy is seen to be essentially at the magnetosheath level, but at its inner edge, the entropy approaches that in the magnetosphere. These facts are consistent with isentropic inflow into the wedge, both from its magnetosheath side and its magnetospheric side, with intermixing occurring only in a narrow layer. The fitted curve used in the reconstruction is also shown in the figure. In the initial data (at $y=0$ ), the pressure is adjusted so that the value of the entropy at all grid points on the $x$-axis is located exactly on this curve. After each step of the integration, the pressure is then nudged so that $S(\psi)$ in fact remains preserved along the streamlines.

The magnetic field map resulting from the MHD reconstruction is shown in the top panel of Fig. 6, in which panel color indicates the values of $B_{z}$. As before, a wedgelike region of reconnected field lines is seen in the map. Its shape is somewhat different from the earlier results and its flux content is slightly larger, requiring an increase of the minimum reconnection electric field from 0.47 to $0.52 \mathrm{mV} / \mathrm{m}$ (we have not redone the reconstruction to incorporate this small increase). The field lines show the presence of a negative normal magnetic field component in most of the magnetopause, in agreement with the negative value, $\langle\boldsymbol{B}\rangle \cdot \hat{\boldsymbol{n}}=-2.3 \mathrm{nT}$, quoted above. The Alfvén speed, based on this field component and a magnetosheath proton density of $10 / \mathrm{cc}$, is $15.2 \mathrm{~km} / \mathrm{s}$. Comparison with the inflow velocity of $13.8 \mathrm{~km} / \mathrm{s}$, mentioned earlier, indicates that, within uncertainties, the inflow is Alfvénic. Indeed, the Walén slope, which is determined, without the use of $\hat{\boldsymbol{n}}$, from data during the traversal of the wedge, is +1.03 (Hasegawa et al., 2004), indicating nearly Alfvénic flow parallel to $\boldsymbol{B}$ in the HT frame. Note that the reconstruction shows an X-point in the upper left corner of the map at $(x, y)=(11500,1400) \mathrm{km}$. The distance from this point to the location of $\mathrm{C} 3$ at the time of its traversal of the reconnection wedge is about $5300 \mathrm{~km}$. The magneto-hydrostatic reconstruction (Hasegawa et al., 2004) exhibited instead an elongated $(3000 \mathrm{~km})$ region in which $\boldsymbol{B}_{\perp} \simeq 0$, located in the same region. This behavior is a likely consequence of the fact that, in the reconstruction frame used by Hasegawa et al. (2004) (the HT frame), the reconnection site was in rapid motion ( $\sim 86 \mathrm{~km} / \mathrm{s}$ toward smaller $\mathrm{x}$-values). In the Teh et al. (2007b) reconstruction, the X-point, if any, was outside the map but would be moving in the same manner.

The second panel in Fig. 6 shows the streamlines obtained from the reconstruction. Because these lines are drawn at constant increments of the mass flux, almost no lines are seen in the magnetosphere, where the plasma density is low. But the white arrows, representing measured flow speeds (transformed to the moving frame) in the reconstruction plane from both $\mathrm{C} 3$ and $\mathrm{C} 1$, show that the velocities seen in the reconstruction frame are larger in the magnetosphere than in the magnetosheath. The reason is that the $\mathrm{X}$-line moves 
(a)

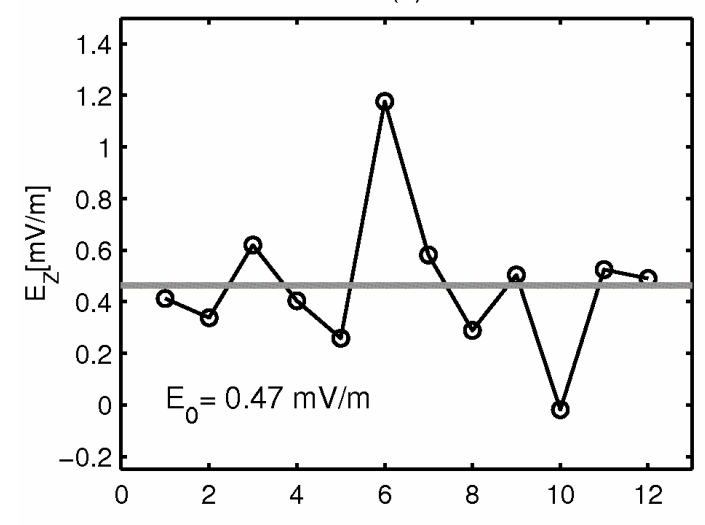

(b)

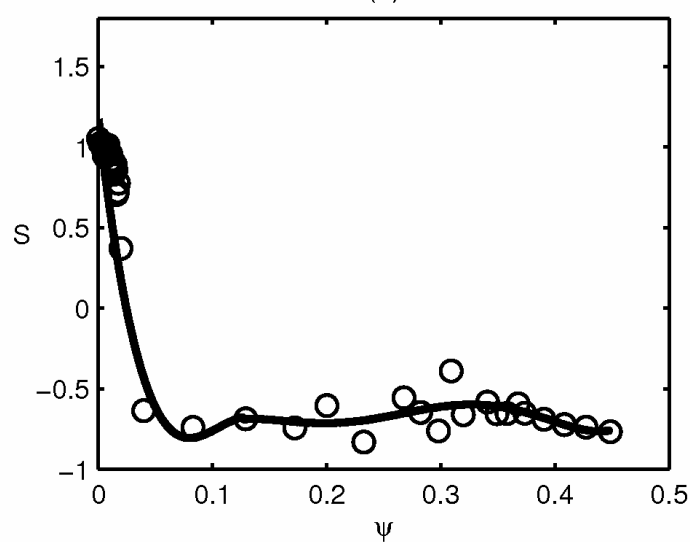

Fig. 5. (a) Plot of axial convection electric field $E_{z}$ for 12 consecutive points in the interval (06:23:53-06:24:37 UT) within the reconnection wedge (between the two green triangles in the first panel of Fig. 6). (b) Plot of the entropy $\mathrm{S}$ versus the stream function $\psi$ and the fitting curve for the reconstruction interval (06:22:00-06:25:21 UT).

downstream at about $140 \mathrm{~km} / \mathrm{s}$, i.e. at nearly the magnetosheath flow speed. The largest velocities are measured by $\mathrm{C} 3$ in the magnetopause itself. They represent the exhaust jet on the sunward/poleward side of the reconnection site. The white arrows are not everywhere precisely parallel to the streamlines as a result of the adjustments, mentioned above, in the component of $\boldsymbol{v}_{\perp}(x, 0)$ perpendicular to $\boldsymbol{B}_{\perp}(x, 0)$.

The third panel in Fig. 6 shows an overlay of field lines (black) and streamlines (white), with the transverse velocity magnitude $\left|\boldsymbol{v}_{\perp}\right|$ in color. In this map, the inflow of plasma from the magnetosheath as well as from the magnetosphere into the wedge of reconnected field lines can be seen, along with the higher-velocity reconnection exhaust, located within the wedge.

The fourth panel again shows magnetic field lines but now with the axial convection electric field, $E_{z}=-(\boldsymbol{v} \times \boldsymbol{B}) \cdot \hat{z}$, in color. As a result of the aforementioned nudging, this electric field component (the reconnection electric field) is constant, except near the reconnection site, where its constancy could not be implemented for numerical reasons, and where it should not remain constant for physical reasons. The total axial electric field should remain constant everywhere but, within a few ion inertial lengths of the reconnection site, it contains, not only the convection field, but also contributions from Hall and perhaps resistive electric fields that are not included in the reconstruction. And, within a few electron inertial lengths from the $\mathrm{X}$, the axial component of the electron pressure tensor, perhaps in combination with resistivity, are the agents responsible for the reconnection electric field. In the region, appearing in purple in the figure, the convection field, $E_{z}$, starts to decrease for numerical reasons as the Xpoint is approached. In a qualitative sense this is the expected behavior. When $E_{z}$ threatens to become negative, which did occur but should not happen in the real case, it was replaced by zero, leading to some errors in the streamline geometry near the X-point. The actual size of the region where ideal MHD becomes invalid must, in reality, be much smaller than the purple region shown in the map.

The fifth panel shows the electric current lines, with the axial current in color. The largest current component in the reconstruction plane is located in the magnetopause and is directed from right to left. It is associated with the large change in $B_{z}$ across the layer and also, via the force $\boldsymbol{j}_{\perp} \times \hat{\boldsymbol{n}}(\boldsymbol{B} \cdot \hat{\boldsymbol{n}})$, with the changes seen in $v_{z}$. The axial current is also concentrated in the magnetopause. Elsewhere, the current density is weak but the current lines exhibit a great deal of small-scale structure.

The sixth panel shows the equipotential lines (red) in the reconstruction plane overlayed on the magnetic field lines (black). One sees that the electric field in the reconstruction plane is directed toward the magnetopause layer on both sides. This behavior causes electric drift in the negative axial $\hat{z}$ direction on both sides of the magnetopause. This motion can be seen in panel two of the figure.

The seventh and eighth panels in Fig. 6 illustrate that the transition in the plasma density and pressure from low to high values occurs slightly outside the earthward boundary of the reconnection wedge.

The four scatter plots in Fig. 7 show, on the upper left, the relation between field components measured by the three other Cluster spacecraft $(\mathrm{C} 1, \mathrm{C} 2$, and $\mathrm{C} 4)$ and those predicted by the C3-based field map. The correlation coefficient in this plot is $c c=0.979$, which should be compared with the earlier results, $c c=0.975$ by Hasegawa et al. (2004) and $c c=0.968$ by Teh et al. (2007b). It is seen that the present result is slightly better but is not as good as the result $(\mathrm{cc}=0.988)$ from the combined optimal map produced by Hasegawa et al. (2005). The upper right scatter plot in Fig. 7 shows the relation between the velocity components measured by $\mathrm{C} 1$ and those predicted by the reconstructed streamline map from $\mathrm{C} 3$. The 

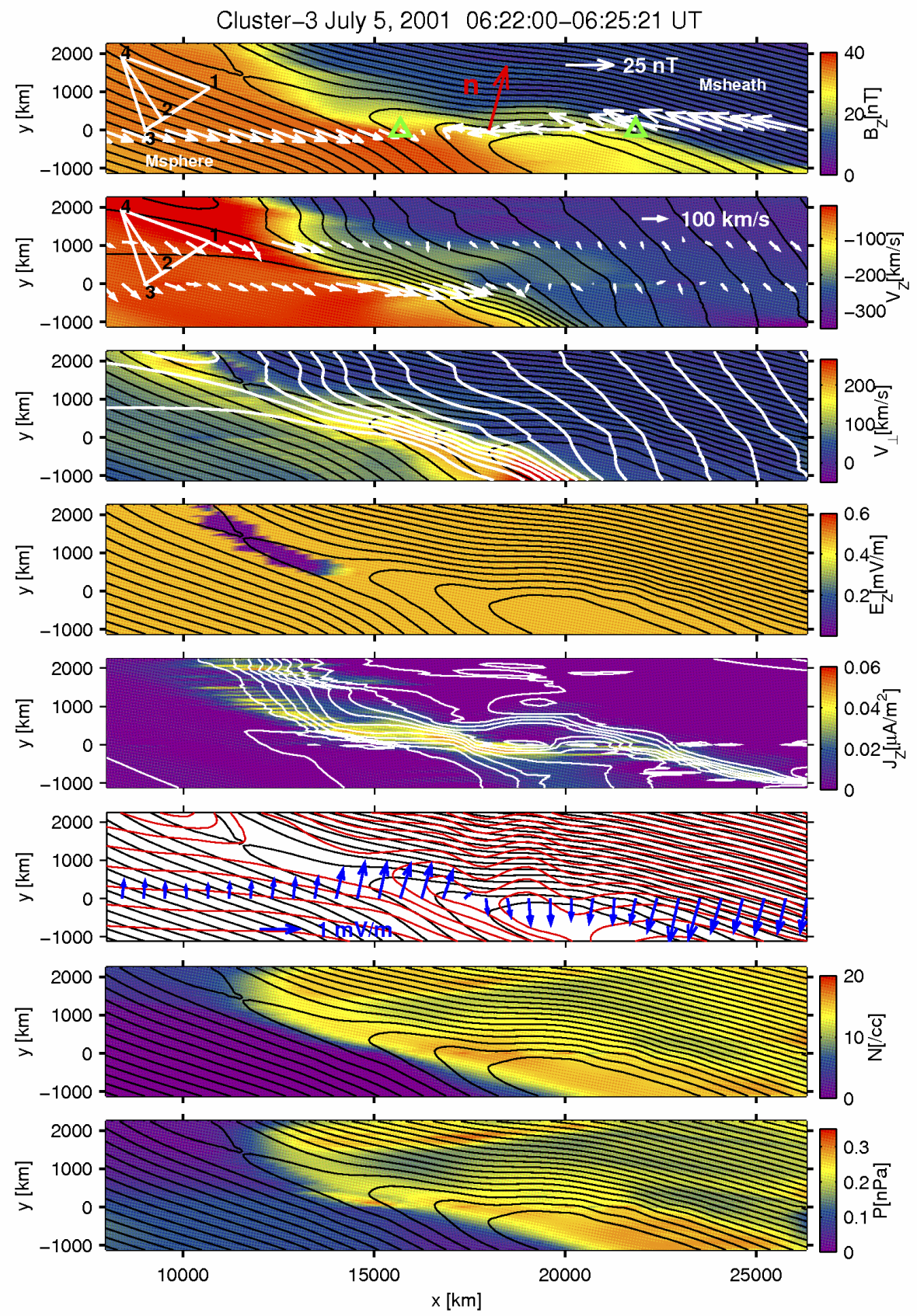

Fig. 6. MHD reconstruction results for the C3 magnetopause crossing on 5 July 2001. The first two panels show a portion of the magnetic field and streamline maps, with the axial magnetic field and axial velocity in color. The third panel is an overlay of magnetic field lines (black) and streamlines (white) with the magnitude of the transverse velocity, $\left|\boldsymbol{v}_{\perp}\right|$, in color. The fourth panel shows the magnetic field lines with the strength of the axial convection electric field in color. The fifth panel shows the in-plane current lines with axial current density in color. The sixth panel shows an overlay of electric equipotentials (red) and magnetic field lines (black), with the in-plane convection electric fields at $y=0$ denoted by blue arrows. The last two panels show the magnetic field map with the ion density and pressure in color. Magnetosphere (magnetosheath) is in lower left (upper right) of the map; sunward/northward is to the right.

correlation coefficient in this plot ( $\mathrm{cc}=0.969)$ is somewhat lower. For the velocity, and also the density and pressure correlations in the bottom two panels, only $\mathrm{C} 1$ data were used because the plasma spectrometer CIS/HIA was func- tional only on $\mathrm{C} 1$ and $\mathrm{C} 3$. The relationship between measured plasma density and pressure at $\mathrm{C} 1$ and corresponding values predicted by the C3 map is less good for two main reasons: (1) The density and pressure levels measured by the 

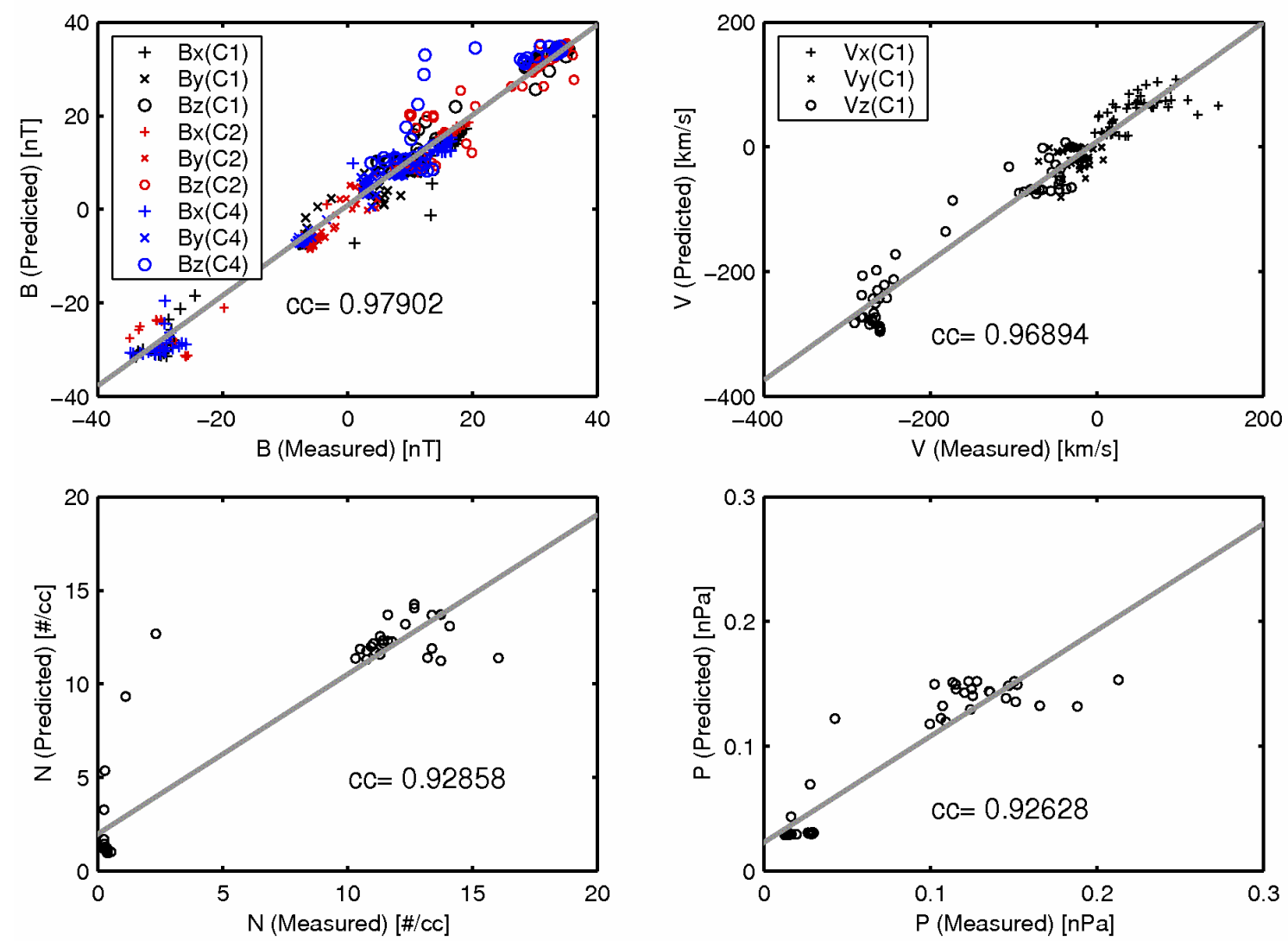

Fig. 7. Comparison of magnetic field components measured by $\mathrm{C} 1, \mathrm{C} 2$, and $\mathrm{C} 4$, and of plasma parameters measured by $\mathrm{C} 1$, with corresponding values predicted by the MHD reconstruction maps created from the $\mathrm{C} 3$ data.

$\mathrm{C} 1$ and $\mathrm{C} 3$ instruments do not agree in the magnetosheath and also not in the magnetosphere; (2) the $\mathrm{C} 3$ map does not predict the center time of the rapid transitions in density and pressure in the $\mathrm{C} 1$ crossing with high accuracy. The extent to which the discrepancies have instrumental origin is not known. But actual time variations must also play a role.

We stress that the correlation coefficient is not an optimal tool for judging the quality of the maps for our event, because the configuration has undergone a substantial change in the time interval between the $\mathrm{C} 4$ and $\mathrm{C} 1$ crossings and the $\mathrm{C} 2$ and $\mathrm{C} 3$ crossings. The poor prediction (see upper left panel in Fig. 7) of $B_{z}$ at points along the path of $\mathrm{C} 4$, the spacecraft furthest away from $\mathrm{C} 3$, may be the result of this temporal evolution. It contributes in a significant way to the lowering of the magnetic correlation coefficient. Finally, we note that the correlation coefficient is not always suitable for the inter-comparison of different events, because, apart from temporal effects, the smaller the separation of the spacecraft, compared to the size of the structure being reconstructed, the higher will be the correlation coefficient.
Table 1. C3 basic parameters for 5 July 2001 event.

\begin{tabular}{lll}
\hline Time interval [UT] & Quantity & Value $^{\mathrm{a}}$ \\
\hline $06: 22: 00-06: 25: 21$ & $\hat{\boldsymbol{x}}$ & $+0.57543+0.21177+0.78996$ \\
& $\hat{\boldsymbol{y}}$ & $+0.42349-0.90347-0.06628$ \\
& $\hat{z}$ & $+0.69967+0.37268-0.60957$ \\
$06: 23: 32-06: 24: 49$ & $\hat{\boldsymbol{n}}^{\mathrm{b}}$ & $+0.56080-0.81509+0.14536$ \\
& $\boldsymbol{V}_{H T}$ & $-236.0-94.5+125.5[\mathrm{~km} / \mathrm{s}]$ \\
& $\boldsymbol{V}_{H T} \cdot \hat{\boldsymbol{n}}$ & $-37.1[\mathrm{~km} / \mathrm{s}]$ \\
$06: 23: 53-06: 24: 37$ & $\boldsymbol{V}_{0}$ & $-80.6-29.6-110.6[\mathrm{~km} / \mathrm{s}]$ \\
& $E_{0}$ & $+0.47[\mathrm{mV} / \mathrm{m}]$ \\
& $\left(\langle\boldsymbol{v}\rangle-\boldsymbol{V}_{H T}\right) \cdot \hat{\boldsymbol{n}}$ & $-13.8[\mathrm{~km} / \mathrm{s}]$ \\
\hline & &
\end{tabular}

a Vector components are GSE.

$\mathrm{b}$ From minimum variance analysis of the magnetic field with constraint $\hat{\boldsymbol{n}} \cdot \hat{z}=0$.

\section{Discussion}

The basic parameters for our event are summarized in Table 1. The reconstruction results for the magnetic field are qualitatively consistent with those obtained from the earlier, GS-based, reconstructions indicating that, in spite of their 


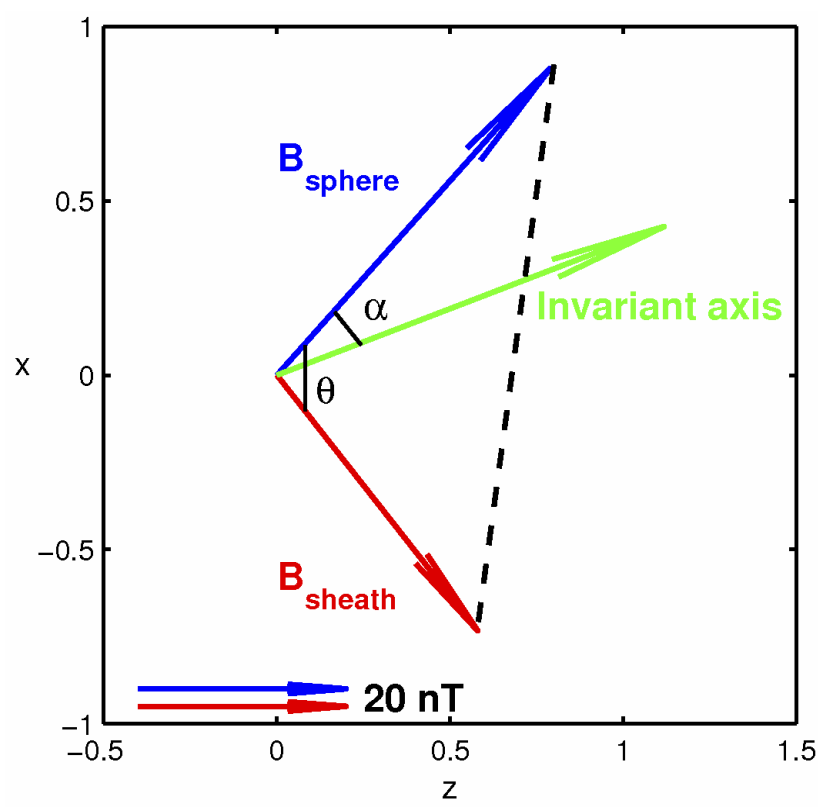

Fig. 8. Schematic, in a plane tangential to the magnetopause, of magnetic fields and invariant axis (X-line). The horizontal (vertical) axis is along the intermediate (maximum) variance direction from MVAB0. The scale is arbitrary. The angle $\alpha$ is $28^{\circ}$ for the axis (green arrow) deduced from the reconstructions (Hasegawa et al., 2004). For the model proposed by Sonnerup (1974), $\alpha=56^{\circ}$, i.e. the axis is perpendicular to the dashed line; the bisector of the angle $\theta$ is at $\alpha=\theta / 2=50^{\circ}$, and the angle predicted by Swisdak and Drake (2007) is at $\alpha=34^{\circ}$.

shortcomings, the GS methods can produce robust results. Onset of reconnection at a proximate site occurred sometime in the time interval between the crossing by $\mathrm{C} 1$ and that by $\mathrm{C} 3$. The average reconnection electric field was at least $0.47 \mathrm{mV} / \mathrm{m}$ (corresponding to a dimensionless reconnection rate of at least 0.025 ) but the shape of the wedge of reconnected field lines in the MHD reconstruction suggests that the configuration obtained from the $\mathrm{C} 3$ data may have been the result of a burst of reconnection at substantially larger reconnection field, leading to the bulge seen at the bottom right of the wedge. It was followed by an interval of lower reconnection rate, producing the narrow tongue of reconnected field lines extending to the X-point in the map. This feature helps produce good correlation between the magnetic fields predicted by the C3-based map (Fig. 6) and the fields measured by $\mathrm{C} 1$ and $\mathrm{C} 4$ prior to the onset of reconnection, when the magnetopause was a thin layer, as shown in Fig. 3.

The orientation and motion of the invariant axis (see Table 1) indicate that the $\mathrm{X}$-line was moving tailward/equatorward along the magnetopause at some 135 $\mathrm{km} / \mathrm{s}$, with the spacecraft on its sunward/poleward side. At the same time the magnetopause itself was moving earthward at about $37.1 \mathrm{~km} / \mathrm{s}$ for a total X-line speed of $140 \mathrm{~km} / \mathrm{s}$. In the field map, the $\mathrm{X}$-point is stationary and located at a distance along the magnetopause of some $5300 \mathrm{~km}$ from the point in the map where $\mathrm{C} 3$ crossed the reconnection wedge. Relative to our new map in the X-line frame, the HT frame, in which the earlier GS-based reconstructions were performed, moves to the right at a speed of about $86 \mathrm{~km} / \mathrm{s}$. In the $60 \mathrm{~s}$ time required for the reconnection wedge to blow past $\mathrm{C} 3$, this velocity gives a displacement of more than $5000 \mathrm{~km}$.

We stress that, in the immediate vicinity of the X-point in Fig. 6, the reconstruction results are not valid because the assumption of ideal MHD breaks down there. Our steady 2$D$ reconstruction method also cannot address the question of how precisely the $\mathrm{X}$-line represents an actual magnetic separator line, connecting two nulls in the full 3-D magnetopause configuration.

The axial magnetic-field component $B_{z}$ (the "guide field") was not the same on the two sides of the magnetopause: As illustrated in Fig. 8, it was strong in the magnetosphere and weak in the magnetosheath. Thus it did not behave as predicted by the traditional model of component reconnection (e.g. Sonnerup, 1974), in which the guide field is the same on the two sides. This early model was based on a comment, made by Petschek (1964), to the effect that, in incompressible MHD, a constant guide field could be added to his basic anti-parallel reconnection geometry without causing it to change. But, later on, Cowley (1976) used an analytical, incompressible MHD model of reconnection to show that the process does not require the guide field to remain constant. Another common assumption is that the $\mathrm{X}$-line should bisect the angle $\theta$ between the field vectors on the two sides so that $\alpha=\theta / 2$. Figure 8 shows that this assumption also does not provide a good prediction of the X-line orientation. Recently, Swisdak and Drake (2007) proposed that the X-line orients itself so as to maximize the flow speed in the reconnection exhaust. Using this hypothesis, and taking into account the density change across the reconnection layer, they developed a simple theory for determination of the angle $\alpha$ in terms of the density ratio, the field magnitude ratio, and the angle $\theta$. As indicated in the figure caption, this prediction is by far the best and falls within $6^{\circ}$ of our invariant axis. However, in reality the orientation of the X-line may not be determined exclusively by local conditions but may also be influenced by the global configuration of flow and field in the magnetopause region. The Swisdak-Drake theory also leads to a value of the maximum exhaust speed in the xy-plane. In our event, their prediction is $227 \mathrm{~km} / \mathrm{s}$, which should be compared to the value $191 \mathrm{~km} / \mathrm{s}$, derived from the measurements (after transformation to the reconstruction frame moving at velocity $\boldsymbol{V}_{0}$ ). The agreement is not perfect but is satisfactory, given the $6^{\circ}$ discrepancy in axis orientation, the simplifications used in the theory, and the time dependence present in the actual event.

The MHD reconstruction shows inflow of magnetosheath plasma into the reconnection wedge and associated acceleration by the magnetic slingshot effect. The flow component measured by $\mathrm{C} 3$ along the direction normal to the 
magnetopause confirms the presence of this flow. One also expects inflow into the wedge from its magnetospheric edge. This flow is also seen in the reconstructed map but, with the exception of one data point, is not present in the normal-flow components, actually measured by $\mathrm{C} 3$ in the magnetosphere. The reason for this discrepancy lies in the small changes of the velocity vectors at $y=0$ that were made prior to the start of the integration in order to obtain a strictly constant value of the axial convection electric field, the assumption being that, at each point on the $\mathrm{C} 3$ trajectory (i.e. at $y=0$ ), the convection field (the axial convection $E_{z}$ ) agrees with the total electric field (the total axial $E_{z}$ ). Possible causes of the difficulties include temporal variations of the magnetopause speed, inaccuracy of the normal vector, or less precise velocity measurements in the low-density magnetospheric plasma. As indicated by the large fluctuations in the measured convective $E_{z}$ that remain in the reconstruction frame (see Fig. 5), it is clear that the reconstructed maps are time aliased as a result of variations, not only in magnetopause speed and orientation, but probably also in $\mathrm{X}$-line velocity and in reconnection rate. Some details of the actual configuration are therefore likely to be smeared out in the reconstruction. However, all of the reconstruction results for this event indicate that the longer duration of the $\mathrm{C} 2$ and $\mathrm{C} 3$ crossings, compared to those by $\mathrm{C} 1$ and $\mathrm{C} 4$ (see Fig. 2) cannot to be explained by a lower magnetopause speed during the $\mathrm{C} 2$ and $\mathrm{C} 3$ crossings than during the $\mathrm{C} 4$ and $\mathrm{C} 1$ crossings (as was attempted by Haaland et al., 2004). The reconstructions show the longer durations to be the result of a thickening of the magnetopause layer caused by a burst of reconnection.

In summary, we have shown that the new MHD-based reconstruction method (Sonnerup and Teh, 2008) is a viable tool for the study of reconnection at the magnetopause, and therefore presumably in other locations and contexts as well (see, e.g. Eriksson et al., 2008 ${ }^{1}$ ). It gives results that are qualitatively consistent with earlier GS-based studies but differs from them in a fundamental way: The reconstruction of a reconnection event is performed, not in the deHoffmannTeller frame, but in a frame of reference moving with the $\mathrm{X}$-line of the reconnection configuration. It is in this frame that a reconnection event should be viewed because, in it, the configuration and the plasma flow are not contaminated by extraneous time variations, caused by translation along the reconnection layer (see Fig. 1). And it is in this frame that the exhaust jets from the reconnection site are most clearly seen. Determination of the orientation and motion of the Xline remains a problem. In principle, they can be obtained (Sonnerup and Hasegawa, 2005) from the requirement that, in a 2-D steady configuration, the total electric field component in the invariant direction (along the z-axis) must be strictly constant, $E_{z}=E_{0}$, i.e. it must be independent of the reconstruction coordinates $\mathrm{x}$ and $\mathrm{y}$. But temporal variations of the motion and of the field geometry often prevent this method from giving meaningful results. This was the case in the event studied here. Fortunately, the z-axis and a reason- able estimate of $E_{0}$ had been established in earlier studies, which allowed the motion of the X-line to be determined.

We emphasize that, at present, our present code does not include dissipation, via resistive effects or plasma mixing, and that it does not accommodate pressure anisotropy. All of these effects are likely to play some role in reconnection events. Resistive effects can be readily included (by use of a one-step spatial lag in the integration) so as to allow for a low-level description of slow-mode MHD shocks. A model to describe entropy generation and other effects caused by mixing would need to be developed in order to include this process. Pressure anisotropy can in principle be included by use of double-polytropic laws (see Appendix of Sonnerup et al., 2006).

The MHD reconstruction presented in our paper shows results, in unprecedented detail, concerning the large-scale structure of a reconnection event at the magnetopause, including maps of magnetic field, flow field, electric current and electric potential distributions, as well as the distribution of plasma pressure and density (see Fig. 6). The reconnection site itself, seen near the edge of the reconstruction domain, is now stationary. In the immediate vicinity of this site, i.e. in the diffusion region, spatial scales comparable to the ion inertial length and smaller are expected to be important but are not included in our reconstruction where, instead, numerical effects allow the reconnection electric field to be maintained in the diffusion region. Streamlines on the ion-inertial scale can be properly described only by including Hall physics, i.e. by using the generalized (two-fluid) form of Ohm's law. We have recently shown that, in principle, reconstruction can be performed also in the two-fluid description, including localized effective resistivity phenomena, if any, (work in progress, Sonnerup and Teh, 2008). Reconstruction at the electron scale is needed at the reconnection site itself. Such reconstruction appears possible as well but remains to be developed in detail.

Acknowledgements. The research was supported by the National Aeronautics and Space Administration under Cluster Theory Guest Investigator Grant NNG-05GG26G to Dartmouth College.

Topical Editor R. Nakamura thanks A. Vaivads and two other anonymous referees for their help in evaluating this paper.

\section{References}

Cowley, S. W. H.: Comments on the merging of non-antiparallel magnetic fields, J. Geophys. Res., 81, 3455-3458, 1976.

Haaland, S., Sonnerup, B. U. Ö., Dunlop, M. W., Balogh, A., Georgescu, E., Hasegawa, H., Klecker, B., Paschmann, G., PuhlQuinn, P., Rème, H., Vaith, H., and Vaivads, A.: Four-spacecraft determination of magnetopause orientation, motion, and thickness: comparison with results from single-spacecraft methods, Ann. Geophys., 22, 1347-1365, 2004, http://www.ann-geophys.net/22/1347/2004/.

Hasegawa, H., Sonnerup, B. U. Ö., Dunlop, M. W., Balogh, A., Haaland, S. E., Klecker, B., Paschmann, G., Lavraud, B., Dan- 
douras, I., and Rème, H.: Reconstruction of two- dimensional magnetopause structures from Cluster observations: verification of method, Ann. Geophys., 22, 1251-1266, 2004, http://www.ann-geophys.net/22/1251/2004/.

Hasegawa, H., Sonnerup, B. U. Ö., Klecker, B., Paschmann, G., Dunlop, M. W., and Rème, H.: Optimal reconstruction of magnetopause structures from Cluster, Ann. Geophys., 23, 973-982, 2005 , http://www.ann-geophys.net/23/973/2005/.

Hasegawa, H., Sonnerup, B. U. Ö., Owen, C. J., Klecker, B., Paschmann, G., Dunlop, M. W., and Rème, H.: The structure of flux transfer events recovered from Cluster data, Ann. Geophys., 24, 603-618, 2006, http://www.ann-geophys.net/24/603/2006/.

Hasegawa, H., Nakamura, R., Fujimoto, M., Sergeev, V. A., Lucek, E. A., Rème, H., and Khotyaintsev, Y.: Reconstruction of bipolar magnetic signature in an earthward jet in the tail: Flux rope or 3D guide-field reconnection?, J. Geophys. Res., 112, A11206, doi:10.1029/2007JA012492, 2007a.

Hasegawa, H., Sonnerup, B. U. Ö., Saito, Y., and Mukai, T.: Recovery of streamlines in the flank low-latitude boundary layer, J. Geophys. Res., 112, A04213, doi:10.1029/2006JA012101, 2007b.

Hau, L.-N. and Sonnerup, B. U. Ö.: Two-dimensional coherent structures in the magnetopause: Recovery of static equilibria from single-spacecraft data, J. Geophys. Res., 104, 6899-6917, 1999.

$\mathrm{Hu}$, Q. and Sonnerup, B. U. Ö.: Reconstruction of magnetic flux ropes in the solar wind, Geophys. Res. Lett., 28, 467-470, 2001.

$\mathrm{Hu}, \mathrm{Q}$. and Sonnerup, B. U. Ö.: Reconstruction of two-dimensional structures in the magnetopause: Method improvements, J. Geophys. Res., 108(A1), 1011, doi:10.1029/2002JA009323, 2003 a.

$\mathrm{Hu}$, Q., Smith, C. W., Ness, N. F., and Skoug, R. M.: Double fluxrope magnetic cloud in the solar wind at $1 \mathrm{AU}$, Geophys. Res. Lett., 30(7), 1385, doi:10.1029/2002GL016653, 2003 b.

Levy, R. H., Petschek, H. E., and Siscoe, G. L.: Aerodynamic aspects of the magnetospheric flow, AIAA J., 2, 2065-2076, 1964.

Lui, A. T. Y., Sibeck, D. G., Phan, T. D., Angelopoulos, V., McFadden, J. M., Carlson, C., Larson, D., Bonnell, J., Glassmeier, K.-H., and Frey, S.: Reconstruction of a magnetic flux rope from THEMIS observations, Geophys. Res. Lett., 35, L17S05, doi:10.1029/2007GL032933, 2008.

Petschek, H. E.: Magnetic field annihilation, in: AAS-NASA Symposium on the Physics of Solar Flares (NASA SP-50), edited by: Hess, W. N., 425-439, 1964.

Shi, Q. Q., Shen, C., Pu, Z. Y., Dunlop, M. W., Zong, Q.-G., Zhang, H., Xiao, C. J., Liu, Z. X., and Balogh, A.: Dimensional analysis of observed structures using multipoint magnetic field measurements: Application to Cluster, Geophys. Res. Lett., 32, L12105, doi:10.1029/2005GL022454, 2005.
Sonnerup, B. U. Ö.: Magnetopause reconnection rate, J. Geophys. Res., 79, 1546-1549, 1974.

Sonnerup, B. U. Ö. and Guo, M.: Magnetopause transects, Geophys. Res. Lett., 23, 3679-3682, 1996.

Sonnerup, B. U. Ö., Hasegawa, H., and Paschmann, G.: Anatomy of a flux transfer event seen by Cluster, Geophys. Res. Lett., 31, L11803, doi:10.1029/2004GL020134, 2004.

Sonnerup, B. U. Ö. and Hasegawa, H.: Orientation and motion of two-dimensional structures in a space plasma, J. Geophys. Res., 110, A06208, doi:10.1029/2004JA010853, 2005.

Sonnerup, B. U. Ö., Hasegawa, H., Teh, W.-L., and Hau, L.-N.: Grad-Shafranov reconstruction: An overview, J. Geophys. Res., 111, A09204, doi:10.1029/2006JA0117, 2006a.

Sonnerup, B. U. Ö., Haaland, S., Paschmann, G., Dunlop, M. W., Rème, H., and Balogh, A.: Orientation and motion of a plasma discontinuity from single-spacecraft measurements: Generic residue analysis of Cluster data, J. Geophys. Res., 111, A05203, doi:10.1029/2005JA011538, 2006b.

Sonnerup, B. U. Ö., Haaland, S., Paschmann, G., Dunlop, M. W., Rème, H., and Balogh, A.: Correction to "Orientation and motion of a plasma discontinuity from single-spacecraft measurements: Generic residue analysis of Cluster data”, J. Geophys. Res., 112, A04201, doi:10.1029/2007JA012288, 2007.

Sonnerup, B. U. Ö. and Teh, W.-L.: Reconstruction of two-dimensional coherent MHD structures in a space plasma: The theory, J. Geophys. Res., 113, A05202, doi:10.1029/2007JA012718, 2008.

Swisdak, M. and Drake, J. F.: Orientation of the reconnection X-line, Geophys. Res. Lett., 34, L11106, doi:10.1029/2007GL029815, 2007.

Teh, W.-L. and Hau, L.-N.: Evidence for pearl-like magnetic island structures at dawn and dusk side magnetopause, Earth Planets Space, 56, 681-686, 2004.

Teh, W.-L. and Hau, L.-N.: Triple crossings of a string of magnetic islands at duskside magnetopause encountered by AMPTE/IRM satellite on 8 August 1985, J. Geophys. Res., 112, A08207, doi:10.1029/2007JA012294, 2007a.

Teh, W.-L., Sonnerup, B. U. Ö., and Hau, L.-N.: Grad-Shafranov reconstruction with field-aligned flow: First results, Geophys. Res. Lett., 34, L05109, doi:10.1029/2006GL028802, 2007 b.

Zhang, Y. C., Liu, Z. X., Shen, C., Fazakerley, A., Dunlop, M., Rème, H., Lucek, E. A., Walsh, A. P., and Yao, L.: The magnetic structure of an earthward-moving flux rope observed by Cluster in the near-tail, Ann. Geophys., 25, 1471-1476, 2007, http://www.ann-geophys.net/25/1471/2007/.

Zhou, X.-Z., Zong, Q.-G., Pu, Z. Y., Fritz, T. A., Dunlop, M. W., Shi, Q. Q., Wang, J., and Wei, Y.: Multiple triangulation analysis: another approach to determine the orientation of magnetic flux ropes, Ann. Geophys., 24, 1759-1765, 2006, http://www.ann-geophys.net/24/1759/2006/. 